\title{
MODELAGEM MATEMÁTICA DA SECAGEM DE CASCA DO FRUTO CAMBUCÁ- PRETO (EUGENIA VELUTINA BERG.) UTILIZANDO DIFERENTES SISTEMAS DE SECADOR
}

\author{
L. G. M. RESENDE ${ }^{2}$, B. S. DOS SANTOS ${ }^{2}$, B. M. TRAVÁLIA ${ }^{2}$, P. B. L. CONSTANT ${ }^{1}$, A. A. C. \\ PAGANI ${ }^{1}$, J. A. B. DOS SANTOS ${ }^{1 *}$ \\ ${ }^{1}$ Universidade Federal de Sergipe, Departamento de Tecnologia de Alimentos \\ ${ }^{2}$ Universidade Federal de Sergipe, Programa de Pós-Graduação em Ciência e Tecnologia de \\ Alimentos \\ *e-mail: joaoantonio@ufs.br
}

\begin{abstract}
RESUMO
O cambucá-preto, pertencente à família Myrtaceae, de espécie Eugenia velutina Berg., é um fruto do cambucazeiro, uma árvore pequena que possui fruto baga vermelho-preta, contendo um caroço porcionalmente, grande envolto em polpa avermelhada, ligeiramente ácida, comestível. A desidratação apresenta a vantagem adicional de colocar ao alcance do consumidor uma maior variedade de produtos alimentícios que podem ser disponibilizados fora da safra. $\mathrm{O}$ estudo de sistemas de secagem pode ser feito por simulação matemática. O objetivo do presente trabalho foi avaliar a curva de secagem da casca do fruto cambucá-preto em dois diferentes sistemas de secagem, ajustadas a seis modelos matemáticos. A secagem foi conduzida em estufa de secagem com lâmpada incandescente e secador de bandejas, ambos com temperaturas de aproximadamente $57^{\circ} \mathrm{C}$. Para obtenção da curva de secagem, realizaram-se pesagens em um intervalo de tempo de 5 minutos durante o período da primeira hora, intervalo de 10 minutos na segunda hora e intervalo de 20 minutos até atingir peso constante. Foram utilizados os modelos matemáticos de Pabis, Newton, Midili, Page, Logarítmico e Wang e Singh. O modelo matemático de secagem proposto por Midilli e por Wang e Singh foram os que melhores se ajustaram aos dados experimentais obtidos na estufa de secagem com luz. Para a secagem feita em secador de bandeja, os modelos de Page e Midilli foram os que melhor representaram a cinética de secagem.
\end{abstract}

\section{INTRODUÇÃO}

Myrtaceae é uma das famílias mais representativas da flora brasileira, principalmente na Mata Atlântica e Restingas (MORI et al, 1983; BARROSO e PERON, 1994; FABRIS e CESAR, 1996; SOUZA e LORENZI, 2005). Esta família inclui árvores ou arbustos com folhas simples, opostas, com glândulas de óleo, que aparecem como pontos translúcidos e margens inteiras. Menezes deLima-Jr. et al. (1997) mostrou que várias espécies da família Myrtaceae exibem atividade anti-inflamatória significativa, associada com óleos essenciais produzidos com estas plantas. Siani e Branquinho (1997) descreveu os óleos essenciais de uma planta como reagentes químicos de defesa contra predadores herbívoros e microrganismos. De acordo com Souza et al. (1998), estes óleos tem um largo espectro de efeitos biológicos, incluindo anticarcinogênico, anti-inflamatória, atividade antiviral e microbicida. 
O cambucá-preto, pertencente à família Myrtaceae, de espécies conhecidas tanto por Eugenia velutina Berg. como Eugenia Macrosperma, é um fruto do cambucazeiro, uma árvore pequena, que pode atingir até três metros de altura. É uma espécie frutífera de ocorrência na Mata Atlântica ou em reflorestamentos no Rio de Janeiro, mas pode ocorrer nos estados vizinhos, em posições de umidade favorável ou que disponham de faixa litorânea quente e úmida. Possui fruto baga vermelho-preta, contendo um caroço porcionalmente, grande envolto em polpa avermelhada, ligeiramente ácida, comestível (PIO-CORRÊA e PENNA, 1926-1978). Na Figura 1 é mostrado a árvore, as folhas e o fruto cambucá-preto.

Figura 1 - Cambucazeiro (A), folhas (B) e fruto cambucá-preto $(\mathrm{C})$.

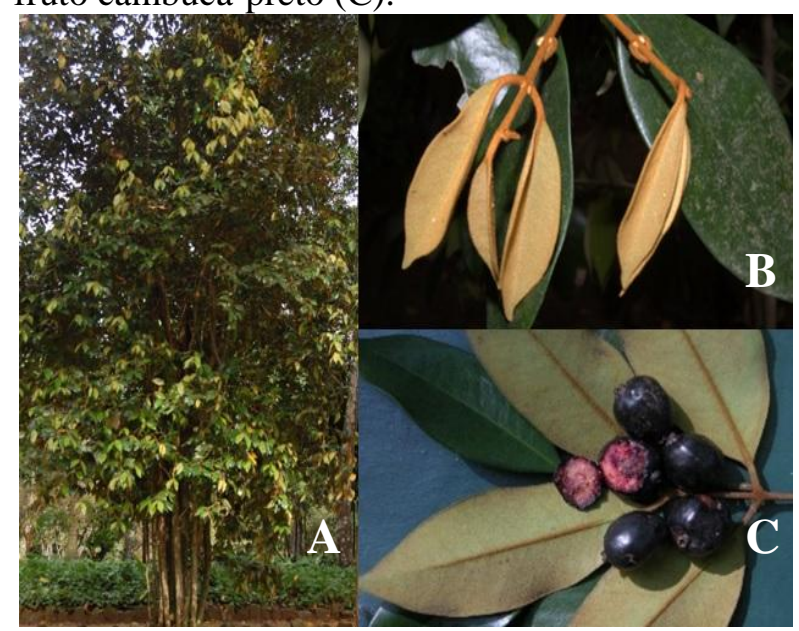

Fonte: E-Jardim (2015)

A forma mais prática de fazer uso industrial de produtos ou insumos alimentícios é desidratando-os. A desidratação apresenta a vantagem adicional de colocar ao alcance do consumidor uma maior variedade de produtos alimentícios que podem ser disponibilizados fora da safra, como é o caso das frutas desidratadas (PARK et al., 2002).

A secagem industrial é a operação pela qual é retirada a umidade contida nos diversos materiais. Trata-se de um fenômeno complexo que envolve simultaneamente a transferência de calor e massa, podendo abranger ainda a transferência de quantidade de movimento. É a operação unitária mais empregada na conservação de alimentos (RIBAS et al, 2000), tanto para o acabamento final ou equilíbrio da umidade própria dos diversos materiais processados com o ar ambiente, como para a sua melhor conservação, como é o caso dos alimentos (COSTA, 2007). Além disso, o processo envolve custos e volumes menores de acondicionamento, armazenamento e transporte.

A obtenção da cinética de secagem é de fundamental importância para a modelagem matemática da operação e para outros fins como projetos de secadores convectivos. A cinética de secagem possibilita, ainda, a determinação do mecanismo predominante na transferência de massa do material para o fluido e as respectivas equações matemáticas correspondentes. O estudo de sistemas de secagem, seu dimensionamento, otimização e a determinação da viabilidade de sua aplicação comercial, podem ser feitos por simulação matemática. Para a simulação, cujo princípio se fundamenta na secagem de sucessivas camadas delgadas do produto, utiliza-se um modelo matemático que representa satisfatoriamente a perda do teor de água do produto durante o período de secagem (JUNIOR e CORREA, 1999).

Diante do exposto, este trabalho teve por objetivo fazer uma avaliação da curva de secagem da casca do fruto cambucá-preto em dois diferentes sistemas de secador, ajustadas a modelos matemáticos.

\section{MATERIAIS E MÉTODOS}

\subsection{Obtenção e Preparo da Amostra}

Foram adquiridos três quilos de cambucá-preto na feira livre do município de Itabaiana - SE. Foram acondicionados em sacos plásticos e, imediatamente, congelados. $\mathrm{O}$ descongelamento foi feito sob refrigeração, 
e, posteriormente, lavados em água corrente e despolpados manualmente a temperatura ambiente, aproximadamente $25^{\circ} \mathrm{C}$.

\subsection{Análise de umidade}

Amostras foram coletadas no início (Tempo 0) e no final do processo de secagem e submetidas ao ensaio de umidade em estufa a $105^{\circ} \mathrm{C}$ até peso constante, em triplicata (IAL, 2004).

\subsection{Cinética de secagem}

Para a secagem foram utilizados dois sistemas, uma estufa de secagem acoplada com uma lâmpada incandescente (100W), o qual atingiu temperatura próxima de $57^{\circ} \mathrm{C}$, e um secador de bandejas com temperatura de aproximadamente $58^{\circ} \mathrm{C}$. Para obtenção da curva de secagem, realizaram-se pesagens em um intervalo de tempo de 5 minutos durante o período da primeira hora, 10 minutos no período da segunda hora e, posteriormente, em um intervalo de 20 minutos até atingir peso constante.

\subsection{Modelagem do processo de secagem}

Os estudos cinéticos foram realizados para determinação das curvas das isotermas de dessorção das cascas dos frutos. Os dados experimentais foram ajustados para as equações empíricas mostradas abaixo, Equações 1, 2, 3, 4, 5 e 6, as quais foram empregadas como correlações para descrever o comportamento de secagem. Estas equações expressam a relação da umidade (RU) em função do tempo.

Modelo de Hederson e Pabis (Henderson e Pabis, 1961)

$R U=a \cdot \exp (-k \cdot t)$

Modelo de Newton (Bruce, 1985)

$R U=\exp (-k \cdot t)$

Modelo de Midilli (Midilli et al., 2002)

$R U=a \cdot \exp \left(-k \cdot t^{n}\right)+b \cdot t$

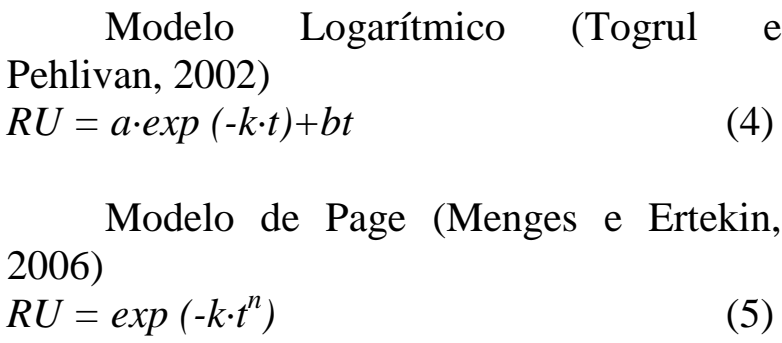

Modelo de Wang e Singh (Wang et al., 2007)

$R U=1+a \cdot t+b \cdot t^{2}$

Onde,

RU = razão de umidade do produto, adimensional;

$\mathrm{t}=$ tempo de secagem, $(\mathrm{s})$;

$\mathrm{k}=$ coeficiente de secagem, $\left(\mathrm{s}^{-1}\right)$;

$\mathrm{a}, \mathrm{b}, \mathrm{n}=$ constantes dos modelos.

Para o cálculo da razão de umidade (RU), utilizou-se a Equação 7.

$R U=\frac{U-U_{e}}{U_{i}-U_{e}}$

Onde,

$\mathrm{U}=$ teor de água no produto, decimal, (bs);

$U_{e}=$ teor de água de equilíbrio do produto, decimal, (bs);

$U_{i}=$ teor de água inicial do produto, decimal, (bs).

\subsection{Análise estatística}

O software Statistica ${ }^{\circledR}$ versão 7.0 para Windows foi utilizado para ajustar as equações dos dados experimentais. $\mathrm{O}$ coeficiente de correlação $\mathrm{R}^{2}$ foi utilizado para descrever o melhor ajuste das equações de cinética de secagem, gerado pelo próprio software estatístico.

O software Assistat versão 7.7 beta, foi utilizado para a realização do teste de Tukey.

\section{RESULTADOS E DISCUSSÃO}

\subsection{Estufa de secagem com luz} incandescente 
Na Tabela 1 são mostrados os coeficientes de determinação $\left(\mathrm{R}^{2}\right)$, obtidos através dos ajustes polinomiais dos resultados experimentais do processo de secagem em estufa com luz da casca do cambucá-preto. A partir das equações empíricas foi possível calcular também os resultados de constante de secagem (k) (Tabela 1).

Tabela 1 - Coeficientes de determinação $\left(\mathrm{R}^{2}\right)$ e constantes $(\mathrm{k})$ referentes às equações $1,2,3,4,5 \mathrm{e}$ 6 , ajustado a secagem de cambucá-preto por secador com luz incandescente.

\begin{tabular}{ccc}
\hline Modelo & $\mathrm{k}$ & $\mathrm{R}^{2}$ \\
\hline Newton & 0,006254 & 0,9881 \\
Page & 0,001622 & 0,9969 \\
Midilli & 0,001538 & 0,9969 \\
Logarítmico & 0,005381 & 0,9938 \\
Wang e Singh & & 0,9969 \\
Henderson e & 0,006750 & 0,9910 \\
Pabis & & \\
\hline
\end{tabular}

Fonte: Autor (2015)

Exceto para o Modelo de Newton, os valores de $\mathrm{R}^{2}$ encontrados permaneceram acima de 0,99, sendo o modelo de Midilli e o de Wang e Singh os que apresentaram maior valor de $\mathrm{R}^{2}$ para o sistema de estufa de secagem com luz. Estes modelos foram considerados os melhores modelos de ajuste, pois quanto maior o $\mathrm{R}^{2}$ melhor é a procedimento de ajuste (TOGRUL; PEHLIVAN, 2004; MAZUTTI et al, 2010;. SANJINEZ-ARGANDOÑA et al., 2011).

$\mathrm{Na}$ condição de secagem, temperatura de aproximadamente $57^{\circ} \mathrm{C}$, considerada pouco drástica, menor $\mathrm{k}$ (constante de secagem) foi observada para os melhores modelos de ajuste. Esta afirmação mostra o quanto os resultados foram coerentes frente a análise do $R^{2}$. Por isso, as equações apresentaram coeficientes de determinação superiores a 99\%, exceto pelo Modelo de Newton, podendo ser utilizadas na estimativa das curvas de secagem de cascas de cambucápreto em secador com luz incandescente.

$\mathrm{Na}$ Figura 2 são mostradas as curvas de secagem dos modelos matemáticos.

A representação gráfica das cinéticas de secagem é similar durante todo o período e em quase todos os modelos utilizados, exceto nos de Pabis e Newton.

Observa-se que a secagem das cascas do fruto ocorre no período de taxa decrescente para a condição estudada, não apresentando taxa período à taxa crescente. Isto é, mostra que a difusão é, provavelmente, o mecanismo físico que governa o movimento da umidade através da estrutura da amostra, ou seja, a velocidade da secagem é controlada pela velocidade de difusão do líquido por meio do sólido, não apresentando um período de taxa constante definido.

A ausência de período à taxa constante foi também observada por Alves (2011), que secou cascas de jabuticabas (Plinia jaboticaba (Vell.) Berg) nas temperaturas fixas de 30, 45 e $60^{\circ} \mathrm{C}$ utilizando um secador de alimentos com cestas metálicas de malha fina.

Figura 2 - Conteúdo de Umidade adimensional (base seca) versus Tempo, ajustado para as Equações de Hederson e Pabis; Newton; Midilli; Logarítmico, Page e Wang e Singh, das cascas de cambucá-preto em secador com luz incandescente.

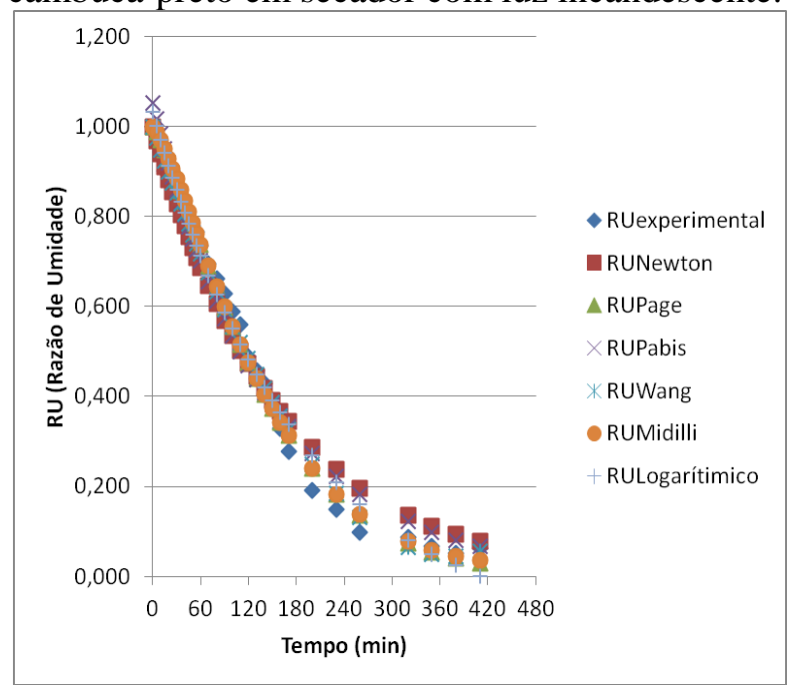

Fonte: Autor (2015) 


\subsection{Secador de bandeja}

Os coeficientes de determinação $\left(\mathrm{R}^{2}\right)$ do processo de secagem em secador de bandeja da casca do cambucá-preto encontram-se na Tabela 2.

Tabela 2 - Coeficientes de determinação $\left(R^{2}\right)$ e constantes $(\mathrm{k})$ referentes às equações $1,2,3,4,5 \mathrm{e}$ 6 , ajustado a secagem de cambucá-preto por secador de bandeja.

\begin{tabular}{ccc}
\hline Modelo & $\mathrm{k}$ & $\mathrm{R}^{2}$ \\
\hline Newton & 0,024344 & 0,9979 \\
Page & 0,014743 & 0,9995 \\
Midilli & 0,015016 & 0,9995 \\
Logarítmico & 0,024003 & 0,9988 \\
Wang e Singh & & 0,9791 \\
Henderson e & 0,025080 & 0,9983 \\
Pabis & & \\
\hline
\end{tabular}

Fonte: Autor (2015)

Para a temperatura, de aproximadamente $58^{\circ} \mathrm{C}$ utilizada na secagem da casca do cambucá no secador de bandeja, verificou-se que, com exceção do modelo de Wang e Singh, todos os outros modelos matemáticos ajustados aos dados experimentais, apresentaram coeficientes de determinação superiores a 0,99 , sendo os modelos de Page e Midilli os de maior valor de $\mathrm{R}^{2}$ e com menores valores de $\mathrm{k}$ (constante de secagem), sendo estes considerados os melhores modelos de ajuste. Para Nunes et al. (2014) realizaram a descrição cinética da fruta jabuticaba em secador de bandeja, o modelo de Page apresentou o melhor ajuste, utilizando como um dos critérios estatísticos de avaliação o maior coeficiente de determinação, concordando assim com o presente trabalho. Em trabalho semelhante, Santos et al. (2010) afirmaram que o modelo de Page foi o que melhor se ajustou aos dados experimentais da secagem da carambola, apresentando maior valor de $\mathrm{R}^{2}$.

As curvas de secagem dos modelos matemáticos estudados encontram-se na Figura 3.

Figura 3 - Conteúdo de Umidade adimensional (base seca) versus Tempo, ajustado para as Equações de Hederson e Pabis; Newton; Midilli; Logarítmico, Page e Wang e Singh, das cascas de cambucá-preto em secador de bandeja.

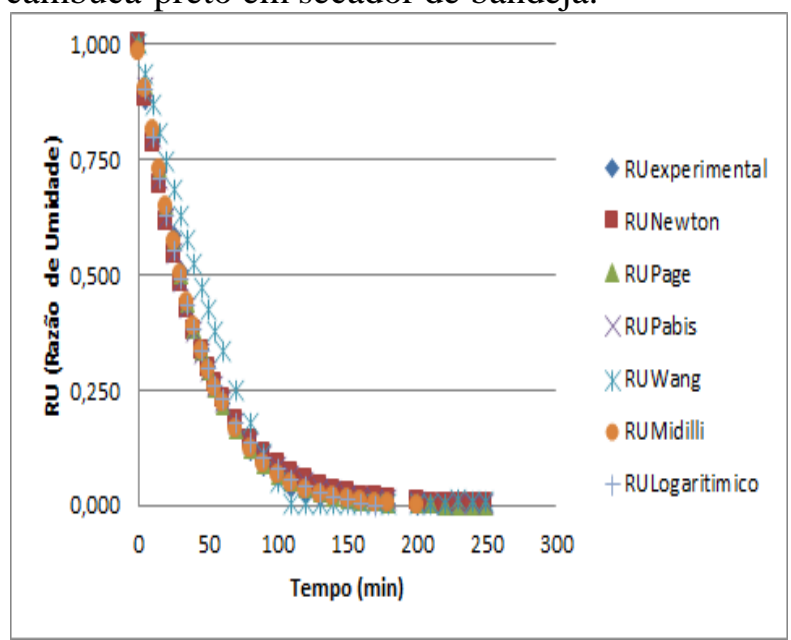

Fonte: Autor (2015)

A razão de umidade reduziu rapidamente no início e, posteriormente, diminuiu lentamente à medida que se aumentava o tempo de secagem. Esse resultado está de acordo com o observado por Santos et al. (2010), ao avaliar as curvas de cinética secagem em termos de razão de umidade e tempo de secagem da fruta carambola em diferentes temperaturas.

Menezes et al. (2013), ao secarem bagaço de maracujá em secador convectivo às temperaturas de $55^{\circ} \mathrm{C}$ e $65^{\circ} \mathrm{C}$, afirmaram que, a estas temperaturas, o bagaço apresentou menor umidade final e menor tempo de secagem.

Por utilizar energia elétrica, este secador tem a vantagem de ser não poluente, apresentando uma elevada eficiência para este processo de secagem.

\section{CONCLUSÃO}


A secagem do cambucá-preto em estufa de secagem com luz incandescente e secador de bandeja foi satisfatória nas temperaturas estudadas, sendo o tempo de secagem inversamente proporcional à temperatura, como o esperado. Os modelos matemáticos de secagem estudados se ajustaram de forma satisfatória aos dados experimentais, sendo o modelo de Midilli (Midilli et al. 2002) o mais adequado para os dois sistemas de secador e na condição de temperatura estudada, por apresentar maior $\mathrm{R}^{2}$ e menores valores de $\mathrm{k}$ (constante de secagem). As equações apresentaram, predominantemente, coeficientes de determinação superiores a $99 \%$, podendo ser utilizadas na estimativa das curvas de secagem de cascas de cambucá-preto em secador com luz incandescente.

\section{REFERÊNCIAS BIBLIOGRÁFICAS}

ALVES, A. P. Casca de jabuticaba (Plinia jaboticaba (Vell.) Berg.): processo de secagem e uso como aditivo em iogurte. Dissertação (Mestrado em Agroquímica), Universidade Federal de Lavras, Minas Gerais, 2011.

BARROS NETO, B.; SCARMINIO, I. S.; BRUNS, R. E. Planejamento e otimização de experimentos. Campinas: Ed. da UNICAMP, 303 p, 1995.

BARROSO, G. M.; PERON, M. V. Myrtaceae. IN: LIMA, M. P. M.; GUEDESBRUNI, R. R. Reserva Ecológica de Macaé de Cima - Nova Friburgo - RJ. Aspectos Florísticos das Espécies Vasculares. Rio de Janeiro: Jardim Botânico, v.1, p. 261-302, 1994.

BRUCE, D. M. Exposed-layer barley drying, three models fitted to new data up to $150^{\circ} \mathrm{C}$. Journal of Agricultural Engineering Research, v. 32, n. 4, p. 337-347, 1985.
COSTA, E. C. Secagem Industrial. Editora Blucher. São Paulo. 2007.

FABRIS， L. C.; CESAR， O. Estudos florísticos em uma mata litorânea no sul do Estado do Espírito Santo. Boletim do Museu de Biologia Melo Leitão 5: p. 15-46, 1996. HENDERSON， S. M.; PABIS， S. Grain drying theory. II: Temperature effects on drying coefficients. Journal of Agricultural Engineering Research, v. 6, n. 3, p. 169174, 1961.

IAL. Normas Analíticas do Instituto Adolfo Lutz. 4ed. São Paulo: IMESP, 1004p, 2004.

JÚNIOR, P.C.A.; CORRÊA, P.A. Comparação de modelos matemáticos para descrição da cinética de secagem em camada fina de sementes de feijão. Revista Brasileira de Engenharia Agrícola e Ambiental, v.3, n.3, p.349-353, 1999.

MAZUTTI, M. A.; ZABOT, G.; BONI, G.; SKOVRONSKI, A.; OLIVEIRA, D.; DI LUCCIO, M.; OLIVEIRA, J. V.; RODRIGUES, M. I.; TREICHEL, H.; MAUGERI, F. Mathematical modeling of thin-layer drying of fermented and nonfermented sugarcane bagasse. Biomass and Bioenergy, v. 34, n. 5, p. 780-786, 2010.

MENEZES-DE-LIMA JR, O.; ROSAS, E. C.; HENRIQUES, M. G. M. O.; BRANQUINHO, L. F.; RAMOS, M. F. S.; SIANI, A. C. Avaliação da atividade antiinflamatória de óleos essenciais de espécies de Myrtaceae e Compositae. III Jornada Paulista de Plantas Medicinais. CPQBAUNICAMP, Livro de Resumos, p. 160, 1997.

MENEZES, M. L.; STRÖHER, A. P.; PEREIRA, N. C.; BARROS, S. T. D. Análise da cinética e ajustes de modelos matemáticos aos dados de secagem do bagaço do 
maracujá-amarelo. Engevista, v. 15, n. 2, p. 176-186, agosto 2013.

MENGES, H. O.; ERTEKIN, C. Mathematical modeling of thin layer drying of golden apples. Journal Food Engineering, v. 77, n. 1, p. 119-125, 2006.

MIDILLI, A.; KUCUK, H.; YAPAR, Z. A. New model for single-layer drying. Drying Technology, v. 20, n. 7, p. 1503-1513, 2002.

MORI, S. A.; BOOM, B. M.; CARVALINO, A. M.; SANTOS, T. S. Ecological importance of Myrtaceae in an Eastern Brazilian Wet Forest. Biotropica, 15: p. 68-70, 1983.

NUNES, J. S.; CASTRO, D. S.; MOREIRA, I. S.; SOUSA, F. C.; SILVA, W. P. Descrição cinética de secagem da polpa de jabuticaba usando modelos empíricos. Revista Verde, v. 9, n. 1, p. 20 -26, jan-mar, 2014.

PARK, K.J.; BIN, A.; BROD, F.P.R. Drying of pear 'd'Anjou' with and without osmotic dehydration. Journal of Food Engineering, v.56, p.97-103, 2002.

PIO-CORRÊA, M.; PENNA, L. A. Dicionário das Plantas Úteis do Brasil e das Exóticas Cultivadas. Rio de Janeiro, Imprensa Nacional, SAI, IBDF: v. 6, 19261978.

RIBAS, A.I.; CÁNOVAS, G.V.B.; GARZA, S.G.; AÑ́, V.G. Métodos experimentales en la ingeniería alimentaria. Zaragoza (Espanha): Acribia, 292 p. 2000.

SANJINEZ-ARGANDOÑA, E. J.; BRANCO, I. G.; BITTENCOURT, T. U.; MUNHOZ, C; L; Influência da geometria e da temperatura na cinética de secagem de tomate (Lycopersicum esculentum). Ciência e Tecnologia de Alimentos, v. 31, n. 2, p. 308312, 2011.
SANTOS, C. T.; BONOMO, R. F.; CHAVES, M. A.; FONTAN, R. C. I.; BONOMO, P. Cinética e modelagem da secagem de carambola (Averrhoa carambola L.) em secador de bandeja. Acta Scientiarum Technology, Maringá, v. 32, n. 3, p. 309-313, 2010.

SIANI, A. C.; BRANQUINHO, L. F. Extração e análise química de óleos essenciais de espécies de Myrtaceae. V Reunião de Iniciação Científica da Fundação Oswaldo Cruz. Anais PIBIC/FIOCRUZ, p. 6, 1997.

SOUZA, M. C.; MENEZES DE LIMA JÚNIOR, O.; ROSAS, E. C.; RAMOS, M. F. S.; SIANI, A. C.; HENRIQUES, M. G. M. O. Efeito antiinflamatório de óleos essenciais de Eugenia jambolana Lam. e Psidium widgrenianum Berg. XV Simpósio de Plantas Medicinais do Brasil. Águas de Lindoia-SP, Brasil, 1998.

SOUZA， V. C.; LORENZI， H. Botânica Sistemática. Nova Odessa: Instituto Plantarum, 2005.

TOGRUL, L. T.; PEHLIVAN, D. Mathematical modeling of solar drying of apricots in thin layers. Journal of Food Engineering, v. 55, n. 3, p. 209-16, 2002.

WANG, Z.; SUN, J.; LIAO, X.; CHEN, F.; ZHAO, G.; WU, J.; HU, X. Mathematical modeling on hot air drying of thin layer apple pomace. Food Research International, Toronto, v. 40, p. 39-46, 2007.

\section{AGRADECIMENTOS}

Os autores agradecem à CAPES, pelo auxílio financeiro concedido por meio de bolsa aos mestrandos do Programa de Pós-Graduação em Ciência e Tecnologia de Alimentos da Universidade Federal de Sergipe. 